\title{
Changes in impedance planimetry metrics predict clinical response to flexible endoscopy in Zenker's diverticulotomy
}

다(1)우우

\author{
Authors \\ Arjun R. Sondhi, Lydia S. Watts, Ryan Law \\ Institution \\ Michigan Medicine, Department of Internal Medicine, \\ Division of Gastroenterology, Ann Arbor, Michigan, United \\ States
}

submitted 10.7 .2020

accepted after revision $\quad 5.10 .2020$

Bibliography

Endoscopy International Open 2021; 09: E31-E34

DOI 10.1055/a-1300-1350

ISSN 2364-3722

(c) 2021. The Author(s).

This is an open access article published by Thieme under the terms of the Creative Commons Attribution-NonDerivative-NonCommercial License, permitting copying and reproduction so long as the original work is given appropriate credit. Contents may not be used for commecial purposes, or adapted, remixed, transformed or built upon. (https://creativecommons.org/licenses/by-nc-nd/4.0/)

\section{Corresponding author}

Arjun Sondhi, University of Michigan, 1500 East Medical Center Drive, Ann Arbor, Michigan, 48109, United States Fax: +1-734-936-7392

rjlaw@med.umich.edu

\section{ABSTRACT}

Background and study aims Various techniques have been described for flexible endoscopic therapy for Zenker's diverticulum (ZD). Objective methods to assess myotomy effectiveness are lacking. We assessed the utility of impedance planimetry in flexible endoscopic ZD therapies and correlation with a validated symptom score.

Patients and methods Patients undergoing endoscopic therapy for symptomatic ZD from February 2019 to March 2020 were included. Intraprocedural impedance planimetry was performed pre- and post-myotomy to assess esophageal diameter and distensibility index (DI). Eating Assessment Tool (EAT)-10 scores were assessed preintervention and post-intervention. Descriptive statistics were calculated.

Results Thirteen patients (46\% women; mean age 80 years; $77 \%$ peroral endoscopic myotomy technique) were included. Technical and clinical success was $100 \%$. No adverse events occurred. At $40 \mathrm{~mL}$ and $50 \mathrm{~mL}$, the diameter improved (mean $2.3 \mathrm{~mm}$ and $2.6 \mathrm{~mm}$, respectively). At 40 $\mathrm{mL}$ and $50 \mathrm{~mL}$, the DI improved (mean $1.0 \mathrm{~mm}^{2} / \mathrm{mmHg}$ and $1.8 \mathrm{~mm}^{2} / \mathrm{mmHg}$, respectively). EAT-10 scores improved by a mean of 15 points. Mean follow-up was 97 days.

Conclusions Intraprocedural impedance planimetry may provide objective data to define success for flexible endoscopic ZD. Further research is required to corroborate these results.

\section{Introduction}

Zenker's diverticulum (ZD) is a rare condition with an unknown overall prevalence given many patients are likely asymptomatic [1]. ZD are pseudodiverticula that manifest as mucosal and submucosal pulsion through Killian's triangle. Symptoms most frequently include dysphagia, regurgitation, and, in severe cases, weight loss and malnutrition [2]. Historically, ZD were treated by an open surgical approach but this has largely been abandoned due to development of transoral interventions using both rigid and flexible endoscopes [2,3].
The conventional flexible endoscopic approach involves complete division of the cricopharyngeal (CP) septum (including the mucosa) to the base of the diverticulum. This approach can be performed using a variety of endoscopic accessories [4]. Recently, Li et al. described a novel technique to divide the $C P$ septum while preserving the mucosa via creation of a short submucosal tunnel. The procedure has been termed Zenker's peroral endoscopic myotomy (POEM) or Z-POEM as the technique is similar to esophageal POEM, an evolving technique in the treatment of achalasia $[5,6]$.

Outcomes following flexible endoscopic intervention for ZD have largely relied on clinical follow-up, which is subjective and 
not standardized. Post-myotomy esophagram findings do not correlate with symptoms [2,7]. With the evolution of Functional Luminal Imaging Probe (FLIP) technology (EndoFLIP, Medtronic, Minneapolis, Minnesota, United States), clinicians are now easily able to objectively measure luminal parameters such as cross-sectional area (CSA), diameter, and distensibility index (DI) using a catheter that is $8 \mathrm{~cm}$ in length and contains 16 sensors within an electrode-containing balloon. The catheter attaches to a console, which executes calculations to perform highresolution impedance planimetry [8]. Impedance planimetry gained initial traction in predicting response to POEM for achalasia, and has been used to evaluate the pylorus in gastroparesis patients undergoing gastric POEM [9]. We aimed to determine if impedance planimetry measurements of ZD improve with flexible endoscopic diverticulotomy and predict clinical response as determined by the previously validated Eating Assessment Tool (EAT-10) $[10,11]$.

\section{Patients and methods}

The study protocol was approved by the Michigan Medicine Institutional Review Board (HUM00160186). Patients were included if all of the following criteria were met: $\geq 18$ years of age, upper esophageal symptoms with compatible esophagram and/or esophagogastroduodenoscopy (EGD) findings demonstrating ZD, and who underwent EGD with impedance planimetry before and after flexible endoscopic CP myotomy. The EAT10 questionnaire was completed by each patient prior to the procedure ( $\downarrow$ Table 1 ). All procedures were performed by a single endoscopist (RL). All patients underwent general anesthesia with endotracheal intubation and placed in the supine position. The impedance planimetry balloon was then positioned across the upper esophageal sphincter (UES) under direct visualization. The balloon was inflated to $40 \mathrm{~mL}$ and $50 \mathrm{~mL}$ for $30 \mathrm{sec}$ onds at each volume and then deflated. Diameter and DI were recorded at each inflation. At the discretion of the endoscopist, the ZD was then treated with a conventional flexible endoscopic CP myotomy or the Z-POEM approach. The decision of which approach to employ was based upon the size of the diverticulum and overall amenability to a POEM approach. A clear cap was attached to the scope and advanced to the diverticulum. In select cases, a transnasal feeding tube was placed into the esophageal lumen to serve as a landmark during division of the CP septum. In the conventional flexible endoscopic CP myotomy approach, an electrosurgical knife was used to incise the CP septum, including the mucosal layer. In contrast, when using the Z-POEM approach, we utilized the same techniques as previously published by Li et al [5]. Prior to hemostatic clip closure of the defect, post-myotomy impedance planimetry data were acquired with the same pre-myotomy methods. The post-procedure EAT-10 questionnaire was obtained within 1 month and then repeated during follow up. Data were analyzed using descriptive statistics.

\section{Results}

Thirteen patients underwent ZD myotomy (mean age 79 years; 6 patients were female). This included one patient previously published as a single case report [12]. Patient demographics, endoscopic approaches, and follow-up time are described in - Table 2. Ten patients (77\%) underwent myotomy via the ZPOEM approach. After the 1-month mark, patients were periodically called to assess for durability of clinical response. Mean follow-up time after the myotomy was 94 days (range 2277 days). No patient reported a recurrence of symptoms. No intra-procedural or post-procedural adverse events (AEs) occurred during the follow-up period. Pre-myotomy impedance planimetry data and EAT-10 scores are described in $>$ Table 3. Post-myotomy data are described in $>$ Table 4 . The changes in impedance planimetry and EAT-10 scores due to the myotomy are described in $>$ Table 5 . All patients reported subjective clinical improvement post-myotomy. Representative pre-myotomy and post-myotomy impedance planimetry topographic readings are shown in > Fig. $\mathbf{1}$ and $\mathbf{D}$ Fig. 2 , respectively. All patients' EAT-10 scores improved, with a mean improvement of 15.3

Table 1 Eating Assessment Tool-10. ${ }^{1}$

To what extent are the following scenarios problematic for you?

My swallowing problem has caused me to lose weight. $\quad 01234$

My swallowing problem interferes with my ability to $\quad \begin{array}{lllll}0 & 1 & 2 & 3 & 4\end{array}$ go out for meals.

Swallowing liquids takes extra effort. 001234

Swallowing solids takes extra effort.

$0 \begin{array}{llll}0 & 1 & 2 & 4\end{array}$

Swallowing pills takes extra effort.

$0 \begin{array}{llll}0 & 1 & 2 & 4\end{array}$

Swallowing is painful.

$0 \begin{array}{llll}0 & 1 & 2 & 4\end{array}$

The pleasure of eating is affected by my swallowing.

$0 \begin{array}{llll}0 & 1 & 2 & 3\end{array}$

When I swallow, food sticks in my throat. $0 \begin{array}{llll}0 & 1 & 2 & 4\end{array}$

I cough when I eat.

$0 \begin{array}{llll}0 & 1 & 2 & 4\end{array}$

Swallowing is stressful.

$0 \begin{array}{llll}0 & 1 & 2 & 4\end{array}$

${ }^{1}$ Adapted from Belafsky [10]

$20=$ no problem. 4 = severe problem.

- Table 2 Patient demographics, endoscopic approaches, follow-up time.

\begin{tabular}{|c|c|c|c|c|}
\hline Patient age, years (mean) & No.female patients (\%) & No.Z-POEM (\%) & No. CP myotomy (\%) & Follow-up time, days (mean) ${ }^{1}$ \\
\hline 54-94 (79.6) & $6(46)$ & $10(77)$ & $3(23)$ & $2-277(94)$ \\
\hline
\end{tabular}


- Table 3 Pre-myotomy impedance planimetry data and EAT-10 scores.

\begin{tabular}{|l|l|l|}
\hline Metric & $\mathbf{4 0} \mathbf{~} \mathbf{L}(\mathbf{m e a n})$ & $\mathbf{5 0 ~} \mathbf{~ L}(\mathbf{m e a n})$ \\
\hline Diameter $(\mathrm{mm})$ & $11-15.7(12.8)$ & $11.3-18.2(13.6)$ \\
\hline $\mathrm{DI}\left(\mathrm{mm}^{2} / \mathrm{mmHg}\right)$ & $1.3-4.4(2.4)$ & $1.1-6.1(2.2)$ \\
\hline EAT-10 (mean) & $7-31(18.3)$ & \\
\hline EAT-10, Eating Assessment Tool. \\
\hline
\end{tabular}

Table 4 Post-myotomy in impedance planimetry data and EAT-10 scores.

\begin{tabular}{|c|c|c|}
\hline Metric & $40 \mathrm{~mL}$ (mean) & $50 \mathrm{~mL}$ (mean) \\
\hline Diameter $(\mathrm{mm})$ & $10.2-19.0(15.2)$ & $10.6-22.0(16.2)$ \\
\hline $\mathrm{DI}\left(\mathrm{mm}^{2} / \mathrm{mmHg}\right)$ & $1.9-12.7(5.1)$ & $1.8-10.5(4.0)$ \\
\hline EAT-10 (mean) & \multicolumn{2}{|l|}{$0-8(3)$} \\
\hline \multicolumn{3}{|c|}{ EAT-10, Eating Assessment Tool } \\
\hline \multicolumn{3}{|c|}{ - Table 5 Change in impedance planimetry data and EAT-10 scores. ${ }^{1}$} \\
\hline Metric & $40 \mathrm{~mL}$ (mean) & $50 \mathrm{~mL}$ (mean) \\
\hline Diameter $(\mathrm{mm})$ & -0.8 to $+3.7(+2.3)$ & -0.7 to $+6.1(+2.6)$ \\
\hline $\mathrm{DI}\left(\mathrm{mm}^{2} / \mathrm{mmHg}\right)$ & $+0.3-7(+1.9)$ & $+0.4-5.3(+1.8)$ \\
\hline EAT-10 (mean) & \multicolumn{2}{|l|}{-31 to $-2(-15.3)$} \\
\hline \multicolumn{3}{|c|}{$\begin{array}{l}\text { EAT-10, Eating Assessment Tool. } \\
{ }^{1} \text { A “+" indicated the value increased or changed to a greater absolute value. } \\
\text { A “-" indicated the value decreased or changed to a lesser absolute value. }\end{array}$} \\
\hline
\end{tabular}

points. The mean diameter increased by $2.3 \mathrm{~mm}$ (-0.8 to 3.7$)$ at $40 \mathrm{~mL}$, which correlated with a mean diameter increase of $18.4 \%(-7.3$ to 31.8$)$. The mean diameter increased by $2.6 \mathrm{~mm}$ $(-0.7$ to 6.1$)$ at $50 \mathrm{~mL}$, which correlated with a mean diameter increase of $18.8 \%$ ( -6.2 to 45.2$)$. The mean DI increased by $1.9 \mathrm{~mm}^{2} / \mathrm{mmHg}$ at $40 \mathrm{~mL}(0.3-7)$, which correlated with a mean DI increase of $71.7 \%(6.8-66.7)$. The mean DI increased by $1.8 \mathrm{~mm}^{2} / \mathrm{mmHg}(0.4-5.3)$ at $50 \mathrm{~mL}$, which correlated with a mean DI increase of $83.9 \%$ (26.7-265). We did not perform impedance planimetry during follow-up. As this was only an exploratory study, we could not justify the additional cost to the patient and minimal risk of performing follow-up impedance planimetry, particularly in patients who were doing clinically well.

\section{Discussion}

To date, no reliable objective intra-procedural marker of success following Zenker's diverticulotomy has been identified. Available data suggest impedance planimetry correlates with outcomes in achalasia after POEM $[13,14]$ and surgical intervention [15]. Based on these findings, we evaluated impedance planimetry technology to assess its predictive ability following flexible endoscopic Zenker's repair.

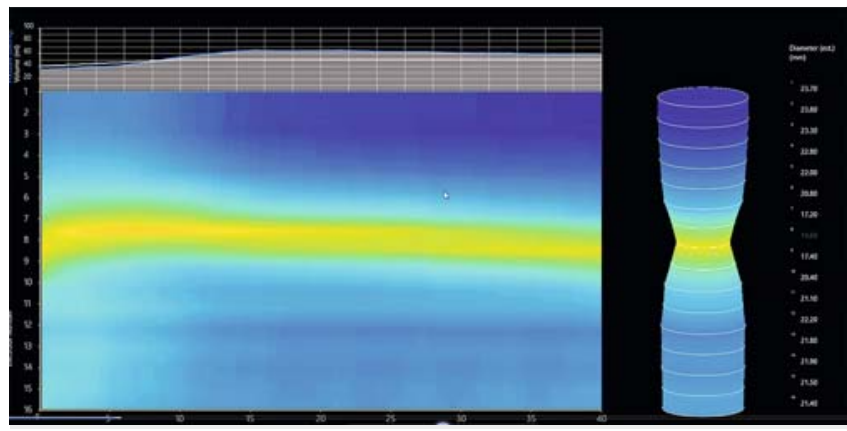

- Fig. 1 Representative pre-myotomy impedance planimetry topographic reading.

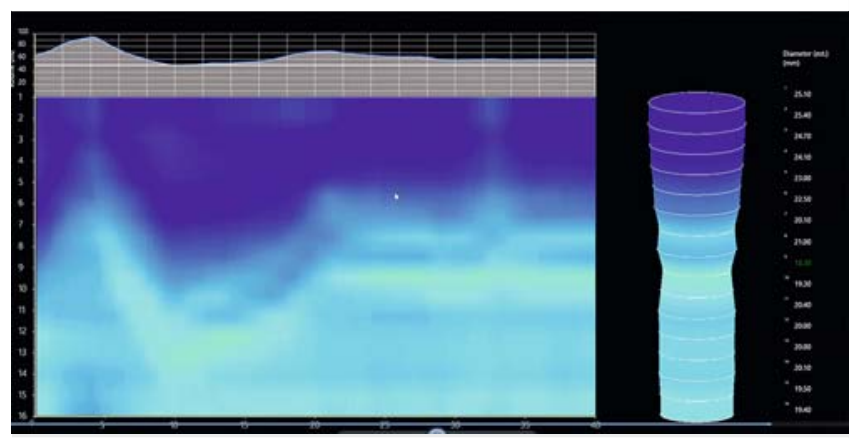

- Fig. 2 Representative post-myotomy impedance planimetry topographic reading.

Our study evaluated 13 patients who underwent successful intervention for the treatment of ZD. All patients demonstrated clinical improvement with endoscopic intervention without AEs. The mean luminal diameter, DI, and EAT-10 all improved. One patient's diameter appeared to decrease given measurements were obtained after hemostatic clip placement, which created a narrowed luminal caliber and likely artifactual decrease in the diameter. In this patient, clip placement was performed prior to impedance planimetry measurements given the patient had an adjacent esophageal diverticulum such that balloon inflation risked increasing the mucosotomy size, potentially resulting in difficult endoscopic closure and tissue apposition.

Notably, the impedance planimetry data and EAT-10 scores improved at $40-\mathrm{mL}$ and $50-\mathrm{mL}$ balloon volumes. We identified that all patients with a DI improvement of at least $26.7 \%$ at $50 \mathrm{~mL}$ experienced a clinical response. This suggests there may be a degree of relative improvement in the DI that could predict clinical success. This is of particular use to the endoscopist as intra-procedural impedance planimetry data could guide when to cease further myotomy. In addition, the percent change between pre- and post-myotomy may be more valuable than a specific diameter, distensibility, or CSA threshold.

In this study, we considered the change in pre-myotomy and post-myotomy impedance planimetry data (i.e. DI, diameter, CSA) to be more valuable than the absolute numbers, as the threshold for technical success when utilizing absolute numbers requires standardized values to characterize normal and 
abnormal. There are limited UES impedance planimetry data available in the current literature, and only in healthy controls. Regan et al. evaluated 13 subjects without any esophageal pathology and used metrics similar to but not the same as DI [16]. Generalizing their data is difficult given balloon volumes were low at $20-\mathrm{mL}$ inflation and correlating manometric data was not obtained. Pyloric and lower esophageal sphincter interventions that utilize impedance planimetry monitoring have noted clinical success based on a change in DI values, even when the post-intervention measurement did not reach the normal value $[9,15]$. This suggests normal values, while important for diagnostic purposes, may not play as important of a role in predicting symptom response to intervention. With these considerations in mind, we believe the percent change between premyotomy and post-myotomy impedance planimetry provides a more suitable parameter to assess technical success.

Several limitations of this pilot study should be noted, including a small sample size from a single tertiary care institution. In addition, all procedures were performed by a single endoscopist. This may limit generalizability. Strengths of this study include prospective approach, maintenance of consistent endoscopic techniques during the myotomy procedures, and use of the validated EAT-10 symptom tool.

Myotomy length was not measured, given that measurements are subjective and likely inaccurate, particularly in Zenker's patients where differences are in millimeters, or centimeters, at best. In addition, identification of the junction of esophageal circular muscle and the cricopharyngeus is difficult to identify in the submucosal tunnel approach. Thus, measurements using a POEM approach may be difficult to interpret. Conversely, in the patients undergoing conventional endoscopic CP myotomy, the risk of AEs may lead to an incomplete myotomy. Thus, the meaning of the actual myotomy length is difficult to interpret. Septotomy length was not compared to DI, given concerns regarding accuracy in measuring septotomy length. Impedance planimetry may be able to guide septum division in the future, but we need to have a reliable target to aim for (i.e. normative post-myotomy data in patients with endoscopically/surgically treated ZD).

\section{Conclusions}

At present, the role of impedance planimetry in patients with ZD remains unclear. The results of our pilot study suggest this technology could be useful in providing objective data. Further research in a larger sample size is required to corroborate these results, evaluate for a possible minimum change in impedance planimetry to achieve clinical response, and compare the efficacy of conventional flexible endoscopic CP myotomy versus the Z-POEM approach.
References

[1] Watemberg S, Landau O, Avrahami R. Zenker's diverticulum: reappraisal. Am J Gastroenterol 1996; 91: 1494-1498

[2] Law R, Katzka DA, Baron TH. Zenker's diverticulum. Clin Gastroenterol Hepatol 2014; 12: 1773-e112

[3] Yuan Y, Zhao YF, Hu Y et al. Surgical treatment of Zenker's diverticulum. Dig Surg 2013; 30: 207-218

[4] Ishaq S, Sultan H, Siau K et al. New and emerging techniques for endoscopic treatment of Zenker's diverticulum: State-of-the-art review. Dig Endosc 2018; 30: 449-460

[5] Li QL, Chen WF, Zhang XC et al. Submucosal tunneling endoscopic septum division: a novel technique for treating Zenker's diverticulum. Gastroenterology 2016; 151: 1071-1074

[6] Yang J, Novak S, Ujiki M et al. An international study on the use of peroral endoscopic myotomy in the management of Zenker's diverticulum. Gastrointest Endosc 2020; 91: 163-168

[7] Mantsopoulos K, Psychogios G, Karatzanis A et al. Clinical relevance and prognostic value of radiographic findings in Zenker's diverticulum. Eur Arch Otorhinolaryngol 2014; 271: 583-588

[8] Hirano I, Pandolfino JE, Boeckxstaens GE. Functional lumen imaging probe for the management of esophageal disorders: expert review from the Clinical Practice Updates Committee of the AGA Institute. Clin Gastroenterol Hepatol 2017; 15: 325-334

[9] Vosoughi K, Ichkhanian Y, Jacques J et al. Role of endoscopic functional luminal imaging probe in predicting the outcome of gastric peroral endoscopic pyloromyotomy (with video). Gastrointest Endosc 2020; 91: 1289-1299

[10] Fernández-Rosati J, Lera L, Fuentes-López E et al. Validation of the eat10 score to detect dysphagia in older people. Rev Med Chil 2018; 146: 1008-1015

[11] Belafsky PC, Mouadeb DA, Rees C] et al. Validity and reliability of the Eating Assessment Tool (EAT-10). Ann Otol Rhinol Laryngol 2008; 117: 919-924

[12] Sondhi AR, Watts LS, Baker JR et al. Functional luminal imaging probe technology to assess response after flexible endoscopic Zenker diverticulotomy. VideoGIE 2019; 4: 551-553

[13] Ngamruengphong S, von Rahden BH, Filser J et al. Intraoperative measurement of esophagogastric junction cross-sectional area by impedance planimetry correlates with clinical outcomes of peroral endoscopic myotomy for achalasia: a multicenter study. Surg Endosc 2016; 30: 2886-2894

[14] Yoo IK, Choi SA, Kim WH et al. Assessment of clinical outcomes after peroral endoscopic myotomy via esophageal distensibility measurements with the endoluminal functional lumen imaging probe. Gut Liver 2019; 13: 32-39

[15] Su B, Callahan ZM, Novak S et al. Using impedance planimetry (EndoFLIP) to evaluate myotomy and predict outcomes after surgery for achalasia. J Gastrointest Surg 2020; 24: 964-971

[16] Regan J, Walshe M, Rommel N et al. New measures of upper esophageal sphincter distensibility and opening patterns during swallowing in healthy subjects using EndoFLIP ${ }^{\circledR}$. Neurogastroenterol Motil 2013; 25: e25-e34

\section{Competing interests}

Dr. Law is a consultant for Olympus America. 